\title{
Polysèmes
}

Revue d'études intertextuelles et intermédiales

\section{Exception et imitation dans Gemma Bovery de Posy Simmonds}

Exception and Imitation in Gemma Bovery by Posy Simmonds

Oriane Monthéard

\section{(2) OpenEdition}

Journals

Édition électronique

URL : http://journals.openedition.org/polysemes/8358

DOI : 10.4000/polysemes.8358

ISSN : 2496-4212

Éditeur

SAIT

Référence électronique

Oriane Monthéard, «Exception et imitation dans Gemma Bovery de Posy Simmonds », Polysèmes [En ligne], 24 | 2020, mis en ligne le 20 décembre 2020, consulté le 21 décembre 2020. URL : http:// journals.openedition.org/polysemes/8358; DOI : https://doi.org/10.4000/polysemes.8358

Ce document a été généré automatiquement le 21 décembre 2020.

Polysèmes 


\title{
Exception et imitation dans Gemma Bovery de Posy Simmonds
}

Exception and Imitation in Gemma Bovery by Posy Simmonds

\author{
Oriane Monthéard
}

1 Dès le premier coup d'œil, Gemma Bovery se présente comme un défi à toute tentative de classification. Indéniablement atypique, cet objet à caractère littéraire et graphique publié en 1999 par Posy Simmonds emprunte à la fois à la bande dessinée et au roman, voire au texte illustré. Il se constitue certes de cases et de strips qui le plus souvent définissent un enchaînement de type séquentiel composant un récit de bande dessinée. Mais ailleurs le texte s'échappe de la vignette et du phylactère pour reléguer l'image au statut d'illustration du récit. Or on admet souvent aujourd'hui que l'image est l'élément du code graphique qui occupe l'espace le plus conséquent sur la planche, et ce serait par elle que passe l'essentiel du sens ${ }^{1}$. Ainsi, plusieurs traits d'écriture tirent Gemma Bovery vers un mode de narration hybride. Tout d'abord, le texte y est particulièrement abondant, dans les bulles, mais surtout sous la forme de commentaires hors case ou de cartouches hypertrophiées dont la calligraphie varie selon la voix qu'elle porte. En parallèle, Gemma Bovery proclame très ouvertement son affinité avec le genre romanesque. La référence affichée à Flaubert s'impose dès le titre et les noms des personnages Gemma et Charlie Bovery, tandis que l'intrigue suit certaines étapes majeures du récit flaubertien. Le recours au narrateur, énonciation assez rare en bande dessinée, affirme aussi l'importance de la dimension textuelle et littéraire, d'autant que la structure narrative choisie est assez complexe, puisque Raymond Joubert, le narrateur qui se veut auteur, présente son ouvrage comme une biographie fabriquée insérée dans un pseudo-journal intime illustré. Le portrait textuel et graphique de Gemma, dont il est l'auteur, se fonde en outre sur des sources diverses dont l'enchevêtrement complexifie le schéma narratif ${ }^{2}$. Une dernière influence se fait enfin sentir dans l'ouvrage, car Posy Simmonds introduit une touche de roman visuel par sa pratique du collage, en intégrant des lettres, schémas, logos, coupures de presse, tickets de transport, copie de tableaux célèbres et extraits littéraires. Gemma Bovery, qui reste dominé par les codes de la bande dessinée, est-il un alors un 
roman graphique, une bande dessinée, un récit dessiné ou une histoire illustrée ? Ces différentes dénominations pourraient certainement toutes correspondre au projet de l'auteur, au genre de l'œuvre et à sa structure protéiforme ${ }^{3}$.

Notons également que le format de Gemma Bovery est en soi une anomalie éditoriale, car il ne reprend ni celui du roman, ni celui de la bande dessinée britannique classique, publiée sous forme de revue, ni celui du roman graphique tel qu'on le connaît depuis les années 1990, qui est beaucoup plus compact. En réalité, le format étroit et allongé de la planche provient des contraintes de maquette imposées par le Guardian, dans lequel le récit a d'abord été publié en 1997 sous la forme d'un feuilleton d'une centaine d'épisodes. D'où ce format tout en hauteur, qualifié de "girafesque " par Paul Gravett $(68)^{4}$, et qui a sans conteste joué sur l'écriture.

3 À première vue, Gemma Bovery s'affiche donc comme une œuvre hors norme et marquée par l'intermédialité. Cette marginalité caractérise cette œuvre à bien des égards, car dans ce récit plein d'humour et de verve, le rapport ambivalent au modèle installe un jeu de superposition et d'inversion entre l'idéal auquel on aspire et le banal qu'il s'agit de fuir, mais aussi entre le particulier et l'universel, jeu qui touche aux sphères sociales, culturelles, littéraires et artistiques. Ainsi Posy Simmonds parvient-elle à décliner et démultiplier une dialectique entre l'exceptionnel et ses envers, tels que le commun, le médiocre, l'insignifiant ou le trivial. Gemma Bovery ne cesse de manipuler la représentation mentale, graphique et textuelle de l'exceptionnel pour la vider de son sens ou la déplacer et la faire réapparaître là où on ne l'attend pas. En parallèle, le traitement satirique de la relation au modèle permet à l'auteur de confirmer l'indépendance de son médium par rapport à l'esthétique romanesque et à d'autres expressions d'une culture idéalisée et vue comme légitime. J'analyserai alors la manière dont texte et image s'associent dans Gemma Bovery pour mettre en place un brouillage entre l'ordinaire et l'exceptionnel, qui désacralise le modèle tout en l'utilisant, en examinant d'abord la construction du héros graphique, qui oscille entre médiocrité et vie d'exception, puis le rapport ambivalent au chef-d'œuvre et enfin la question de la distinction culturelle dans le roman graphique.

\section{Splendeurs et misères du personnage graphique}

4 Tout au long de ce roman graphique, le destin des héros est une affaire d'image(s) constamment remise(s) en cause par la satire. À un premier niveau, l'intrigue retrace le parcours d'un couple qui cherche à porter son destin au-delà de l'ordinaire, même si l'attention se concentre plus particulièrement sur Gemma et ses aspirations secrètes. Un bref synopsis permettra de mieux saisir les enjeux de la reconstitution biographique dont l'hérö̈ne fait l'objet. Ainsi, Gemma souffre de sa rupture avec Patrick, son grand amour de jeunesse, mais se console dans les bras de Charlie, qu'elle épouse rapidement. Après quelque temps à Londres, où le couple mène une vie médiocre dans un logement peu confortable, il décide de s'expatrier en Normandie, cette terre vue comme exotique, où, pense-t-il, les attend une vie plus captivante. Une fois installée dans leur longère normande, et passé l'enthousiasme initial, Gemma ressent les premiers symptômes de l'ennui. Elle rencontre Hervé de Bressigny, qui devient son amant mais se lasse d'elle assez vite, et elle meurt peu de temps après la rupture. Imiter la vie à la française pour échapper à une vie londonienne sans fard est donc un échec et ne mène qu'à la désillusion puis à la mort. Pour donner chair à cette trame narrative, 
Posy Simmonds place l'image au cœur de l'opposition entre l'ordinaire et l'exceptionnel dans la construction du personnage. Elle joue en même temps sur les différents sens de l'image en employant celle-ci à la fois comme un élément constitutif de la planche en bande dessinée, comme une représentation mentale (jusqu'à l'illusion) et comme une représentation sociale et collective potentiellement trompeuse.

5 La fausse image est ainsi matérialisée d'un point de vue graphique, Gemma Bovery exploitant ces différentes fonctions de l'image pour figurer les représentations erronées dont les personnages sont tour à tour auteurs et victimes. La vie dont rêvent Gemma et Charlie n'est en effet qu'un ensemble d'idées préconçues qui prennent la forme d'une projection picturale, ou la copie ratée de ce qu'ils voient comme l'essence de la vie à la française. C'est ce qu'on lit dans les pensées que Joubert prête à Gemma avant qu'elle ne vienne s'installer en France et que ce dernier définit comme «a bourgeois fantasy » (33) : «Every night she repainted the picture : a life of rustic bliss over the Channel... the wine, the food, the veg they would grow, the work they would do, the friends who would stay, the baby they might have... » (30). Pour souligner la naïveté de cette représentation déjà verbalement picturale, Joubert traduit l'idée par le style de l'image, car le fantasme du tableau parfait se meut en coloriage pour enfant (figure 1):

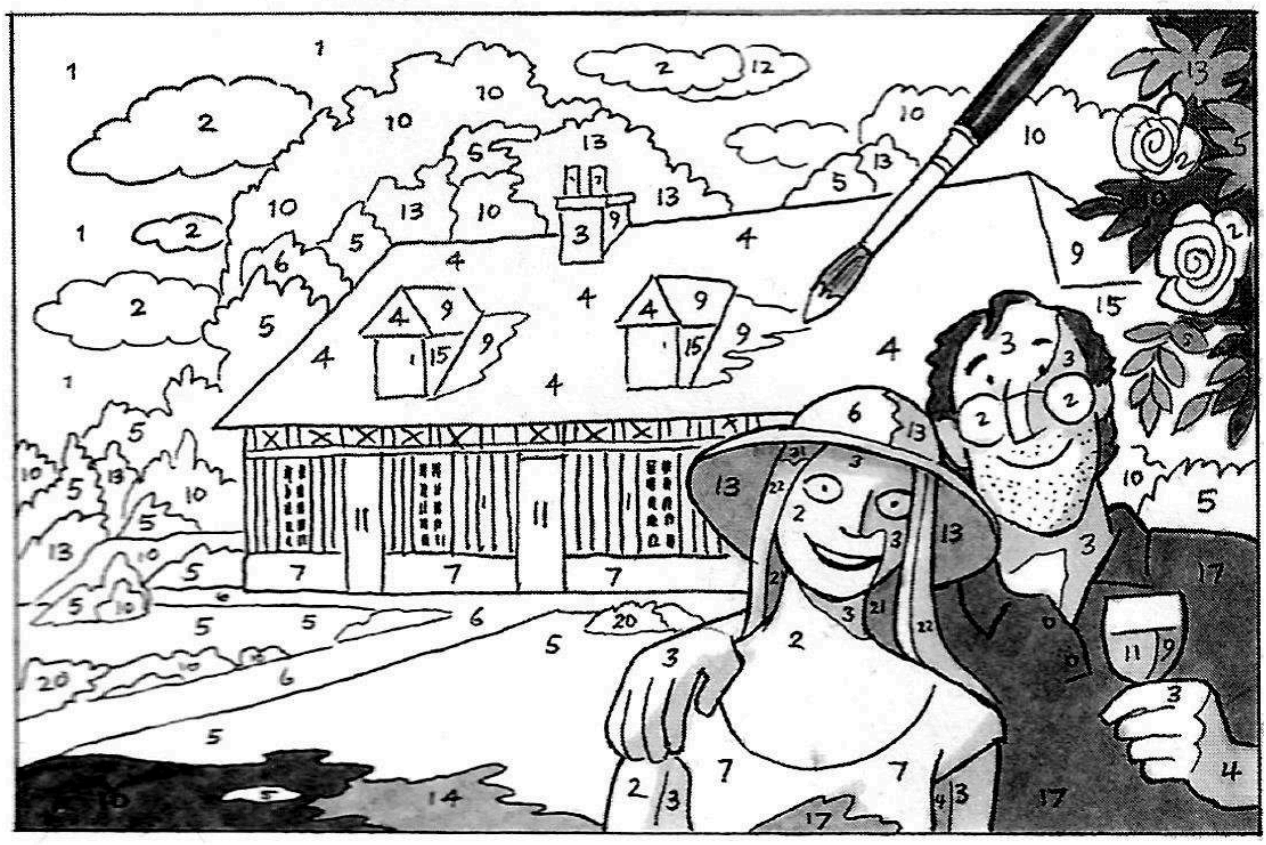

Figure 1. Posy Simmonds, Gemma Bovery. London: Jonathan Cape, 1999, p. 30.

6 Une fois installé en Normandie, Charlie revêt quant à lui tous les signes extérieurs, et donc graphiquement représentables de son idéal de vie à la française. Endossant tous les stéréotypes, il s'affuble du costume du Français campagnard tel qu'un Britannique est censé se le représenter : avec sa barbe de trois jours, il arbore souvent un béret et un marcel à la propreté douteuse, fume des gauloises tout en transportant sa baguette fraîche sous le bras et se régale de café au calvados dans le bar PMU du village. On le voit, le personnage porte sur lui une construction mentale collective éculée et devient en cela une caricature (figure 2) : 


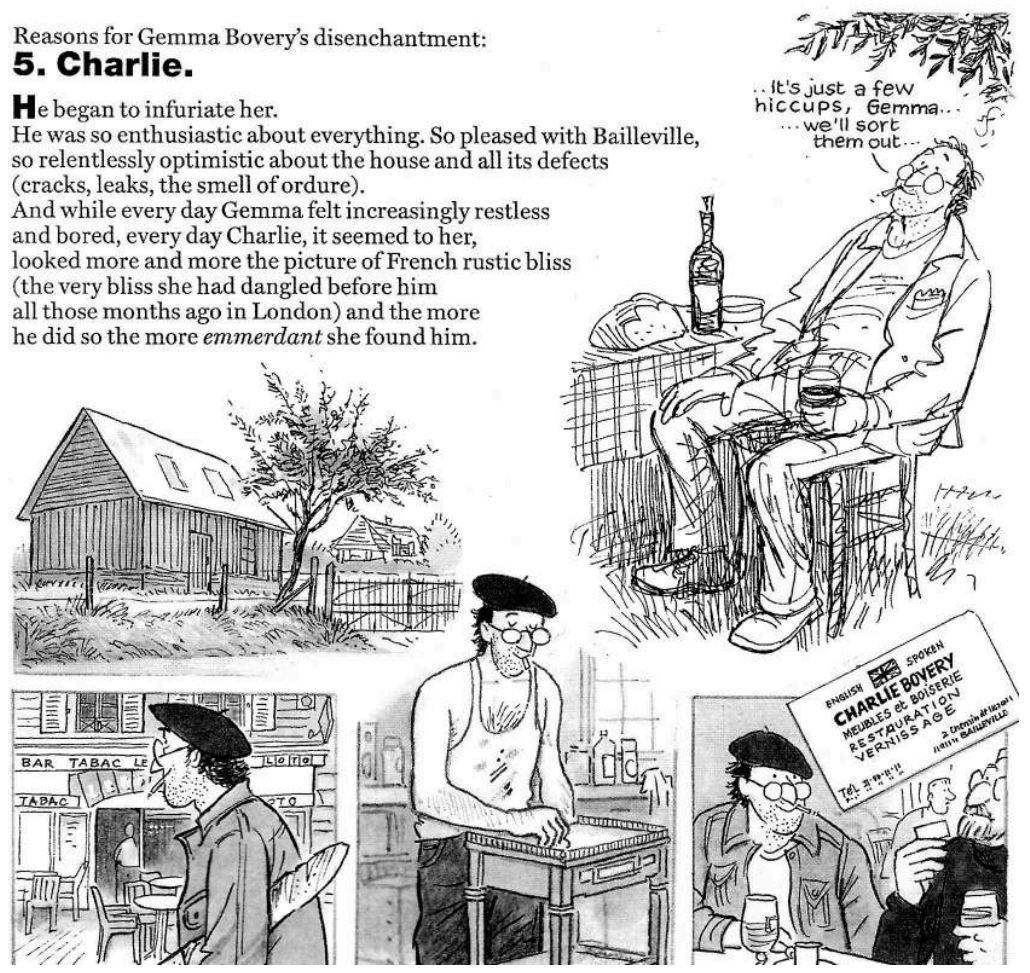

FiguRE 2. Posy Simmonds, Gemma Bovery. London: Jonathan Cape, 1999, p. 40.

7 Un autre exemple est apporté par les tentatives de Gemma pour se hisser hors de la médiocrité en travaillant sur son apparence (puisque la vie à Bailleville, nom qui suggère l'ennui, n'a finalement rien d'exceptionnel). Maîtriser sa silhouette, redécorer son intérieur, peindre: tous ces efforts, qui sont les manifestations visibles et parfaitement graphiques d'une tentative de progrès, visent à l'amélioration de son statut par l'image. Ironiquement, en cherchant à s'élever, elle ne fait pourtant qu'imiter des modèles connus et renforcer les clichés.

Cette banalité du personnage au cœur de la satire est également visible dans la corporéité, et donc, dans le trait qui façonne les personnages. Souligner les défauts physiques par le dessin est une des techniques dont use souvent la dessinatrice dans ses dessins de presse, qui est le format d'origine de Gemma Bovery, et dans ses autres publications, comme True Love, Tamara Drew ou Cassandra Darke. Dans Gemma Bovery, elle parvient à dessiner la banalité à travers une certaine indétermination, touchant non pas les visages, qui sont parfaitement différenciés, mais le corps, dont le contour est souvent informe. Elle entoure et enveloppe les silhouettes sans les marquer, dans des étoffes lâches, des vestes et manteaux amples et ce, parfois même dans les scènes qui se déroulent dans les intérieurs. Un autre trait courant dans cette construction graphique va dans le même sens : Joubert ainsi que Charlie Bovery sont fréquemment dessinés le dos courbé ou avachis dans un fauteuil, une gestuelle qui selon les moments représente l'accablement et la dépression, ou bien l'inertie d'un personnage passif et peu capable de construire son destin.

9 Ce sera particulièrement vrai dans le cas de Charlie, dont la présence physique évoque un relâchement de la chair et de l'ossature, un flou dans le geste et l'attitude qui correspondent ici à un personnage sans relief. Cette indétermination graphique signale la transparence du personnage d'un point de vue à la fois social et esthétique. Font 
exception les moments où il est représenté en train de restaurer les meubles, art dans lequel il excelle. À chaque fois le corps se tend dans des poses plus maitrisées, voire gracieuses, parfois en un mouvement presque dansé, soudain gratifié d'une valeur esthétique qui montre à quel point le talent est à même d'élever le personnage.

Le corps de Gemma fait l'objet d'un traitement particulier. Dans la première partie du récit, le vêtement ample dissimule ce corps semble-t-il sans forme, comme dans la vignette ci-dessous (figure 3) :

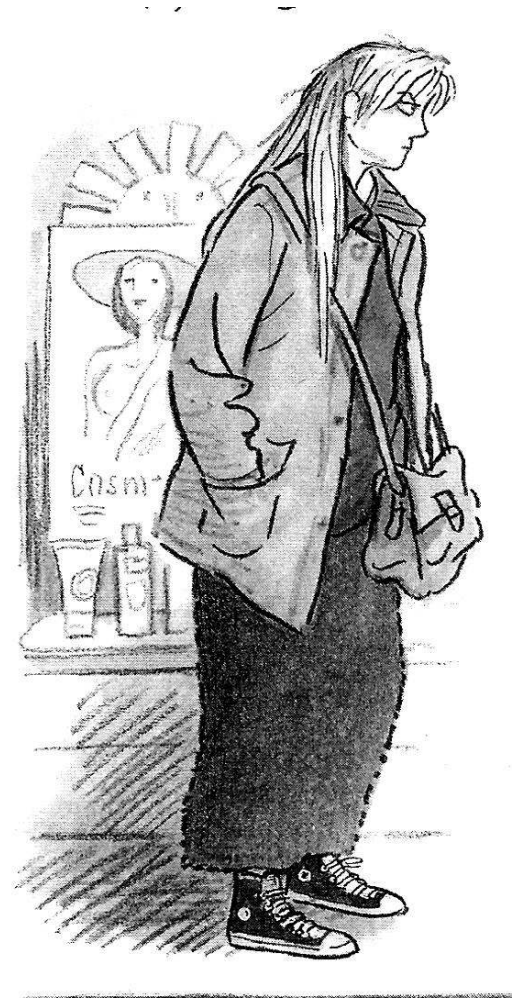

Figure 3. Posy Simmonds, Gemma Bovery. London: Jonathan Cape, 1999, p. 39

Mais plus tard, pour accompagner l'évolution de l'héroïne, le corps de celle-ci devient plus visible, partiellement découvert et même érotisé. En effet, peu après son arrivée en France, elle s'efforce d'améliorer son apparence négligée et lutte pour redessiner les contours de sa silhouette; son style vestimentaire s'affirme, tout comme le trait qui la dessine. De la lingerie dont elle se défait dans les scènes amoureuses, on peut percevoir les détails finement ciselés, contrairement aux formes vagues et matières semble-t-il plus grossières qui l'habillaient auparavant. La métamorphose ainsi produite se traduit par un dessin plus ferme, et les contours du corps tout comme les traits du visage, désormais soulignés, rendent le personnage plus remarquable tout en le différenciant des autres (figure 4) : 


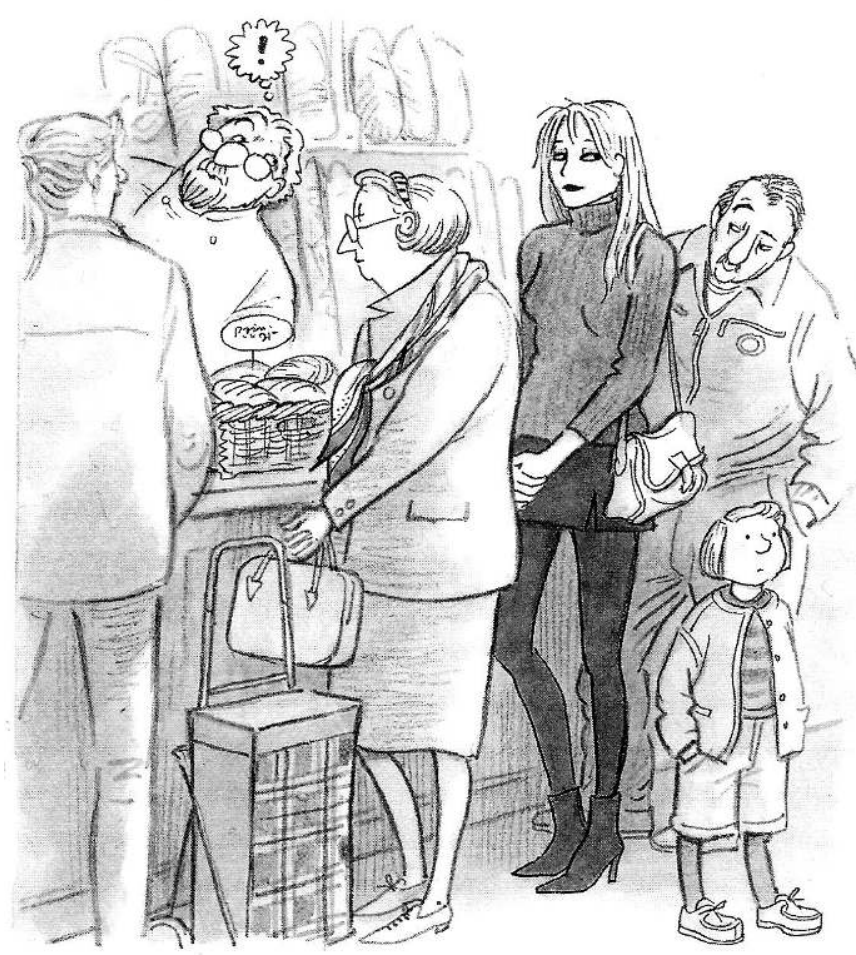

FiguRE 4. Posy Simmonds, Gemma Bovery. London: Jonathan Cape, 1999, p. 59 dèle littéraire, qui les rend uniques. Comme le lecteur du roman graphique, le narrateur est d'abord frappé par leur nom où résonne le texte de Flaubert. Aussi, le caractère littéraire de la vie de Gemma est établi dès la première planche, lorsque Joubert qualifie son histoire de tragédie. Plus tard, c'est parce que leur échec, celui du couple et de celui de l'installation en France, rappelle la vie ratée de Charles et Emma qu'ils deviennent personnages littéraires, parce que l'association avec le canon fonctionne, du moins en partie. Toute la démarche d'écriture entreprise par Joubert vise alors à faire de Gemma un sujet et un personnage littéraires, et donc une représentation. Tout au long de sa narration, son travail d'écrivain consiste à structurer et organiser le récit d'une vie, afin que chaque étape coïncide avec celles du texte flaubertien: il analyse scrupuleusement les causes de l'ennui de Gemma et les classe par ordre inverse de priorité, réagence les événements et reconstitue le fil biographique, applique sur l'expérience censée être vécue le vernis de la fiction et la rend ainsi remarquable.

Cette transfiguration du destin par le traitement littéraire de l'expérience est presque simultanément mise à mal par la vision satirique qui traverse le roman graphique : ainsi, au début du récit, l'admiration soudaine de Joubert pour ses voisins, l'aura dont ces derniers bénéficient grâce à l'association avec Flaubert, est relativisée par le malentendu comique consistant à associer Gemma et son combi Volkswagen qui 
semblent alors exercer sur Joubert la même fascination. De même, la remarque du narrateur après la première rencontre est extrêmement ironique, d'autant qu'il s'agit d'un commentaire a posteriori, à un moment où l'échec est connu: «Nestling in its green embrace, their little house, with smoke curling from the chimney resembled the last scene of a fairy tale, the page where the protagonists live happily ever after » (33). Le commentaire est habilement placé en bas de la planche, pour créer l'effet classique du cliffhanger, tout en l'utilisant à contre-emploi, puisque l'histoire ne prend pas ce chemin. L'auteur nous prévient ainsi que l'association du personnage à la sphère littéraire n'a rien de fixe ou d'objectif. C'est aussi la soif d'exception qui est objet de satire puisque c'est elle qui rend les personnages ordinaires. Mais c'est également par leur banalité qu'aux yeux du narrateur ils imitent la destinée littéraire de personnages existants, et qu'ils sont en cela, et paradoxalement, extirpés du lot commun. Au-delà de l'intrigue et de la construction des personnages, le lien avec le texte d'exception qu'est l'œuvre canonique structure Gemma Bovery et permet à Posy Simmonds de jouer avec le concept et la pratique de l'imitation littéraire.

\section{Le rapport au chef-d'œuvre, de la transposition à la digestion par l'image}

Posy Simmonds se donne en effet l'occasion à la fois d'exploiter les effets de l'imitation et d'en ébranler certains codes. Ce type de démarche n'est pas resté unique dans sa carrière: l'intrigue de Tamara Drew, publié en 2007, est empruntée à Farfrom the Madding Crowd tandis que Cassandra Darke, publié en 2018, revisite A Christmas Carol. À chaque fois, Posy Simmonds pratique l'imitation, ce "phénomène [...] général qui consiste à s'inspirer d'un autre texte pour écrire le sien »(Decout 5), mais elle y superpose l'adaptation, puisque les textes premiers sont transposés vers un autre médium. Dans Gemma Bovery, le lien au chef-d'œuvre prend d'abord la forme d'une transposition vers le code graphique par des emprunts divers qui s'assument et s'exhibent, puisque le roman graphique calque sa trame narrative sur celle de Madame Bovary et multiplie les références au texte de Flaubert. Les points communs sont flagrants : l'intrigue est fondée sur la désillusion et l'ennui de l'héroïne qui poussent celle-ci à l'adultère, avec en arrière-plan une peinture sociale féroce de la Normandie profonde. Toute la lecture d'Yvan Leclerc, spécialiste de Flaubert, va dans ce sens. Selon lui, on retrouve dans Gemma Bovery non seulement le scénario global du roman classique, mais également un rythme similaire grâce aux alternances entre des épisodes de pur récit et des moments plus descriptifs. Il remarque aussi que Posy Simmonds transpose subtilement le thème flaubertien de l'imitation, à travers l'ambition sociale des personnages et le rapport presque maladif aux objets. La fidélité au modèle serait donc narrative et même formelle sur certains points (Leclerc 50-51). On trouve de nombreux parallélismes et équivalences notés aussi par Danièle Wargny: Charlie restaure les meubles, tandis que Charles répare les corps malades. À la scène du fiacre chez Flaubert correspond l'épisode du combi Volkswagen garé dans un parking de Rouen. Charles a eu une première femme, comme Charlie; dans les deux récits se retrouvent dès le départ les problèmes financiers du couple et plus tard la douleur du veuf. Le narrateur est boulanger, probablement en référence au premier amant d'Emma. Outre les noms des deux personnages principaux de Simmonds, n'oublions pas celui de Joubert, où résonne en dernière syllabe le nom de l'écrivain français. Enfin, 
pour Danièle Wargny, le $\mathrm{G}$ ajouté à « Emma " pourrait bien être une discrète référence à Gustave (209-218). Ces équivalences sont cependant loin d'être systématiques, et la transposition est délibérément inégale.

Par ailleurs, cette adaptation passe par la « dégradation » appuyée du chef-d'œuvre, car le roman graphique opère une transformation parodique et burlesque qui affiche son décalage avec Madame Bovary. Outre de nombreux éléments de l'intrigue qui éloignent les deux œuvres et dont certains sont liés au changement d'époque ou de structure narrative (Gemma a étudié aux Beaux-Arts; Charlie est divorcé; le narrateur tombe amoureux de Gemma, etc.), les différences de traitement et de tonalité sont évidentes car le registre est essentiellement comique et satirique. La langue est souvent oralisée, se fait parfois grossière, et fait l'objet de divers travestissements comiques, comme lorsque le français est massacré par les Anglais peu soucieux de s'intégrer ou qu'à l'inverse les Français, hormis le narrateur, ânonnent un anglais approximatif. Aussi Gemma Bovery s'ancre-t-il volontiers dans le prosaïque, sans doute en une translation contemporaine de l'écriture réaliste. C'est notamment le cas dans la planche qui recense les divers défauts de la maison normande, source de grande déception pour Gemma. La longère est en effet vétuste, attaquée par les moisissures et infestée de vermine tandis que la tuyauterie défaillante diffuse en permanence une odeur d'égout. Le dénouement, enfin, est grotesque car Gemma ne meurt pas de désespoir, comme on pourrait s'y attendre, mais s'étouffe avec un morceau de pain confectionné par le boulanger narrateur. Posy Simmonds force ainsi le trait, et les effets comiques de ces transpositions font de ce récit en images une copie volontairement déclassée du récit de référence et ce, à des fins satiriques qui font de lui autre chose qu'une simple « mise en cases » du roman classique.

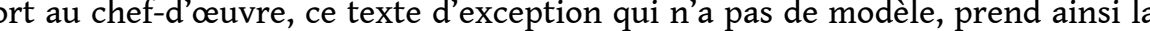
forme d'une transformation assez insolente. Pourtant, quand elle aboutit au roman graphique ou à la bande dessinée, l'adaptation du texte littéraire signifie souvent soumission au genre source dans une hiérarchisation implicite des valeurs, les ressorts graphiques se faisant les plus discrets possible et le texte adapté se devant de prévaloir. Faire de l'adaptation un acte éminemment irrespectueux en malmenant le texte modèle, voilà comment Posy Simmonds parvient à ébranler ces classifications tout en éludant la question de la hiérarchie entre les genres. L'approche comparative, si elle reste utile pour déterminer les modalités d'imitation ou d'adaptation et la nature du lien avec le texte premier, n'est donc pas suffisante. Il faut aussi considérer ce roman graphique issu de l'adaptation en tant que création en soi et comme point de départ de l'analyse ${ }^{5}$. Il semble justement que Posy Simmonds n'utilise pas le langage de la bande dessinée pour se hisser vers le canon littéraire et en offrir une version modernisée en images, mais qu'elle procède inversement: elle s'emploierait principalement à "s'empar[er] du thème de l'imitation tel que l'a construit Flaubert pour le soumettre au discours critique de la bande dessinée » (Garric). Ainsi, l'abondance du texte dans Gemma Bovery, inhabituelle en bande dessinée, n'a pas été dictée en premier lieu par un désir de s'inscrire dans la filiation de l'écriture romanesque mais plutôt par des contraintes éditoriales imposées par le Guardian : afin de contenir l'intrigue dans cent épisodes, Posy Simmonds a dû s'appuyer sur davantage de texte pur ${ }^{6}$. À ce titre, elle a conçu Gemma Bovery d'abord comme une bande dessinée en tant que telle avant d'y absorber l'intrigue de Flaubert. De la même façon, le personnage dessiné de Gemma provient probablement des différents travaux de Posy Simmonds dans les années qui 
précèdent et des « réservoirs de personnages » (Gravett 67) que constituent ses carnets de croquis. Dans le processus d'adaptation mis en œuvre ici, le médium et ses contraintes ainsi que la pratique de l'auteur semblent concurrencer le texte adapté comme moteur de la création. De manière générale, le projet d'écriture de Posy Simmonds dans Gemma Bovery consiste probablement autant à produire une adaptation de Madame Bovary qu'à représenter certains travers des Français et des Anglais à des fins comiques. N'oublions pas que Gemma Bovery a d'abord été conçu pour la presse et que sa vocation était satirique. Posy Simmonds visait alors un public britannique qui ne pouvait qu'être sensible à ces portraits proches de la caricature.

Tout en tissant des liens avec le chef-d'œuvre de Flaubert, Gemma Bovery s'écarte aussi du schéma classique de l'adaptation vers la bande dessinée dans la mesure où son auteur utilise le procédé pour en faire un sujet en soi, notamment en travaillant sur l'image de Madame Bovary et celle de son héroïne. Tout d'abord, le souvenir du roman, de son intrigue et de son héroïne hante Gemma Bovery par le biais du narrateur et de sa connaissance intime du texte classique. Le roman et ses personnages apparaissent à travers le filtre de la perception personnelle et fort inventive de Joubert, ce qui permet de faire de l'imitation un élément de l'intrigue : le procédé s'intègre à la fiction pour devenir un «fantasme de gens lettrés » (Garric). Aussi le procédé de l'imitation, comme le rappelle Maxime Decout, est-il parfois comparé à une digestion ${ }^{7}$ plus ou moins bien menée, idée que Posy Simmonds semble prendre au pied de la lettre et manier avec ironie. En effet, est-ce un hasard si Joubert, en tant que narrateur et auteur, exprime régulièrement ses émotions par l'état de son ventre qui se mue alors en organe parlant? Ainsi, au début de l'œuvre, la souffrance physique qu'il ressent depuis la mort de Gemma est le symptôme d'un sentiment de culpabilité qui le pousse à écrire et vient justifier sa prise de parole : «My head tells me I am merely at fault, but my guts condemn me. How I suffer--my colonic agony--it's not just à cause d'une colite. [...] However, I do not examine these events to soothe my digestion. I do it for my own conscience, for the sake of my sanity " (2). Joubert est par ailleurs celui qui digère les événements comme les textes, celui de Flaubert en priorité, mais aussi les carnets de Gemma qu'il savoure, recycle et ressert au lecteur selon sa recette personnelle. Notons enfin que Jean-Luc Fromentin, éditeur français de Gemma Bovery, aurait dit de Posy Simmonds, qu'elle « réécrit finalement toute l'intrigue en "cannibalisant" le texte littéraire » (Servin 96), ce qui suggère que le processus de transposition ainsi opéré allie l'hommage à la transformation profonde et à l'incorporation du texte classique dans les modalités du roman graphique. Il faut en même temps souligner que la présence de Flaubert dans le récit n'est presque jamais langagière, que Joubert ne s'approprie jamais ses mots pour le pasticher et le cite peu, hormis à travers la lettre de rupture extraite du roman qu'il envoie à Gemma de la part de son amant. Le récit est ainsi un produit très transformé résultant d'une assimilation du texte par le narrateur, qui le conduit à interpréter l'expérience d'autrui à partir de sa mémoire du chef-d'œuvre. À travers lui, et peut-être en un geste réflexif, Posy Simmonds rend compte de la façon dont le texte canonique peut agir non seulement sur une autre œuvre en termes d'inspiration, mais aussi en tant que présence mémorielle dans l'esprit de l'écrivain sous la forme d'une véritable emprise.

19 L'action du chef-d'œuvre sur l'esprit créateur est donc de l'ordre de l'idée, mais aussi de l'image encore, dans son sens littéral cette fois. Deux fois dans le récit, la mémoire de l'autre texte se manifeste par une représentation mentale et visuelle. Lorsque certaines situations rappellent à Joubert le texte de Flaubert, surgissent sur la planche 
ces portraits qui imitent et détournent le style de gravures du XIX ${ }^{\mathrm{e}}$ siècle censées représenter la célèbre héroïne, sous la forme d'une intertextualité plastique. Dans l'image ci-dessous où s'embrassent les amants, le cadre du miroir apparemment creux peut se voir comme le lieu de passage entre fiction et réalité, entre expérience et interprétation de l'expérience. La référence au mythe de Narcisse, quelque peu détourné puisque le personnage embrasse le reflet de son amant et non le sien, vient en outre réaffirmer la tension entre le même et l'autre (figure 5) ${ }^{8}$ :

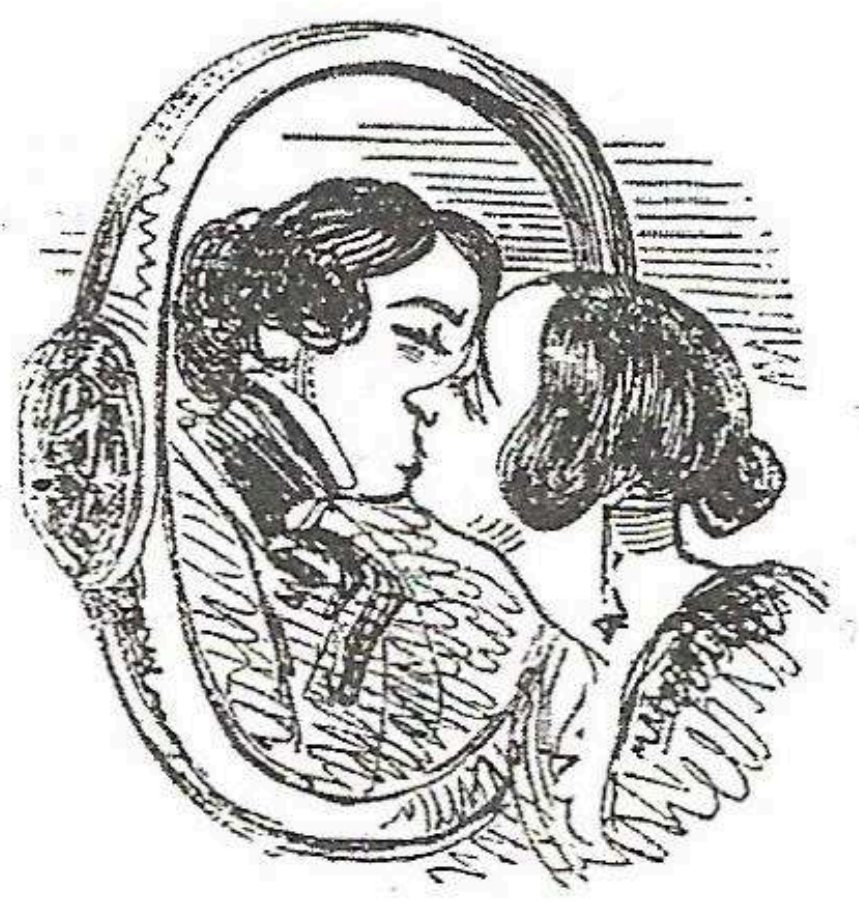

FiguRE 5. Posy Simmonds, Gemma Bovery. London: Jonathan Cape, 1999, p. 46

Le texte littéraire ainsi présent à travers une image emblématisée se retrouve comme absorbé par la mise en page de la bande dessinée. En même temps, ces apparitions, qui semblent émaner directement de l'esprit du narrateur, en disent long sur celui-ci et son lien au texte. Au moins autant qu'une façon de faire correspondre les intrigues, le récit est l'histoire d'une obsession littéraire quelque peu pathologique, la juxtaposition entre les deux visages dans les vignettes ci-dessous suggérant même la quasi-identification du narrateur-auteur avec son personnage, dans un clin d'œil à la déclaration que l'on attribue couramment à Flaubert, « Madame Bovary, c'est moi » (figure 6) : 
If Gemma had committed adultery, she gave nothing away at dinner. There were none of the usual signs - no guilty over-attentiveness to her spouse; none of those smiles and blushes that arrive unbidden with some steamy recollection. No, Gemma wore her usual cool expression. (Fishes look at you with more animation.) I began to think I was wrong. Nothing had happened. She was probably a prude and, in any case, she didn't look the part of an adulteress - or certainly not the literary one I had in mind. (Exquisite, dark, refined, yet possessed of a fulminant sexuality.)

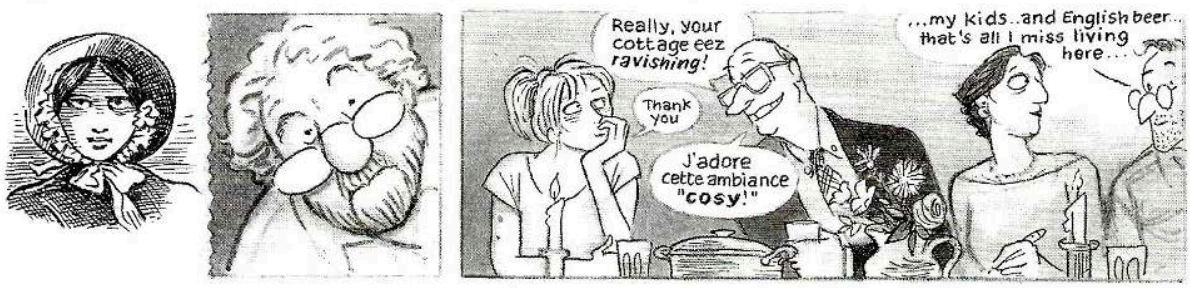

Figure 6. Posy Simmonds, Gemma Bovery. London: Jonathan Cape, 1999, p. 50.

Un commentaire du narrateur tente pourtant de nier ce rapport malsain au modèle au moment même où la gravure s'invite sur la planche :

[...] I remember feeling a strange glee. It meant that Hervé and Gemma might meet again. I confess my imagination had raced ahead: I already saw them in naked embrace. Something which made me laugh out loud. The absurdity of it. Life imitating Flaubert's masterpiece: Madame Bovary crosses the path of the local squire, Rodolphe, just as Gemma, a few minutes ago, had crossed Hervé's. Well, I say "just as", but it was of course nothing like the novel - Life rarely imitates Art. Art has some point to it, whereas Life... (46)

L'ironie du passage tient au fait que toute la construction narrative de Joubert consiste justement à faire correspondre artificiellement la vie avec l'œuvre d'art, à lire dans le vécu ordinaire de ses voisins celui certes non moins médiocre d'Emma et Charles, mais qui reste le destin d'icônes littéraires. On goûtera également la façon dont Joubert balaie le principe wildien d'un revers de la main, tout en y adhérant à travers la citation ainsi que la démarche globale du récit dessiné. Il est bien un lecteur wildien de Flaubert, mais aussi de l'expérience, car il lit dans la vie des autres comme dans un livre'. On le voit, Posy Simmonds se préoccupe autant d'imiter le chef-d'œuvre en le détournant et en le ramenant à ses préoccupations que de manipuler le procédé de l'imitation. Pour preuve, vers la fin du récit, le texte de Flaubert n'est plus seulement une référence ou une source d'inspiration à l'intérieur du récit, il devient agent de l'intrigue, car Joubert envoie à Gemma une copie de la lettre de rupture entre Rodolphe et Emma, et lui laisse croire que c'est son amant qui cherche à rompre. L'extrait devient même une arme dans les mains de Joubert: "I went immediately to my study and seized the weapon, a new copy of Madame Bovary » (72). Enfin, peu avant l'épilogue, et au moment où le narrateur renoue les fils de l'intrigue, le roman est considéré moins comme une source dans le travail d'écriture qu'un objet déclencheur malfaisant: « Tears fill my eyes and I weep. For Gemma, for the waste of her life, and for Charlie, for his heart, which I think is broken. I reach behind me for a handkerchief. Is it by chance that my hand falls not on the box of Kleenex, but on Gemma's copy of Madame Bovary? (103). Ainsi, tout en ayant recours à l'adaptation en tant que procédé, Posy Simmonds l'utilise également pour en faire un ressort parodique, tout en définissant d'autres liens graphiques avec le roman. Cet usage de l'adaptation et ces diverses manières de détourner le chef-d'œuvre contribuent à la peinture de l'environnement culturel et artistique dans Gemma Bovery et conduisent Posy Simmonds à explorer les liens de son roman graphique avec la culture classique. 


\section{La distinction culturelle dans Gemma Bovery}

Dans Gemma Bovery, la connaissance et la culture prennent une place importante dans l'intrigue et dans la construction d'un narrateur qui cherche à se distinguer socialement. Le regard de Posy Simmonds sur la question alimente la satire sociale grinçante, mais lui permet également d'explorer la notion de culture généralement pour en souligner toute la relativité ${ }^{10}$. Joubert est le personnage qui porte l'idée de l'ascension sociale par le savoir, car il est l'intellectuel qui a lu et ingéré non seulement Flaubert mais aussi bien d'autres auteurs, se dit cultivé malgré sa vie simple et se déclare écrivain. Cette auto-proclamation fait l'objet d'un traitement satirique car son talent artistique est minimisé par un passé peu glorieux en la matière (ses écrits sur les Francs-maçons à Rouen pendant la Révolution semblent assez obscurs et n'ont finalement pas été publiés). Posy Simmonds aborde aussi la relativité de cette notion de culture par la comparaison entre les Français et les Anglais et la perception différente qu'ils peuvent avoir des objets culturels. Ainsi, les Normands qui connaissent Flaubert ou au moins le titre de son roman le plus connu, s'amusent tous de l'homophonie avec les noms des deux Londoniens, fait auquel Gemma reste insensible : " everyone from the bloody baker upwards comments on Madame Bovary, it's so boring » (41).

Chez Gemma, la recherche d'un absolu, ou au moins celle d'un accomplissement, passe aussi par la conquête culturelle, mais prend la forme d'une imitation assez passive et présentée comme une faute de goût. Alors qu'elle cherche à s'insérer dans ce qu'elle perçoit comme un idéal culturel, elle se contente de copier les modèles préexistants. Ainsi, lorsqu'elle se remet à la peinture, elle ne cherche qu'à reproduire la nature, puis mime le geste de Monet. Joubert souligne la banalité de ce projet: «Normandy was, after all, the great outdoor atelier of the nineteenth century. How could not one be reminded of those giants of paintings - Millet, Monet etc? » (38). En même temps, dans la vignette représentant Gemma en train de chercher l'inspiration, l'idée est relayée par l'intégration d'une copie des Meules de Monet, dans une transposition dessinée du chef-d'œuvre. La juxtaposition, de l'ordre du montage, l'écart de talent supposé entre les trois artistes que sont Monet, Gemma et Joubert (voire les quatre, si on envisage un geste d'autodérision de la part de Posy Simmonds) et l'effet d'immobilité ainsi produit soulignent avec humour combien la comparaison peut sembler artificielle (figure 7) :

Outside was equally disturbing: Gemma claims that when she went out sketching the landscape somehow immobilised her. After a while she couldn't draw. Anything.

This doesn't surprise me. Normandy was, after all, the great outdoor atelier of the nineteenth century. How could one not be reminded of those giants of painting- Millet, Monet, etc? How could one not feel inhibited, one's efforts absolutely pigmy? So on the day when she described herself sitting hunched at the edge of a harvest field, it was perhaps the thought of Monet's Haystacks that froze her drawing hand.
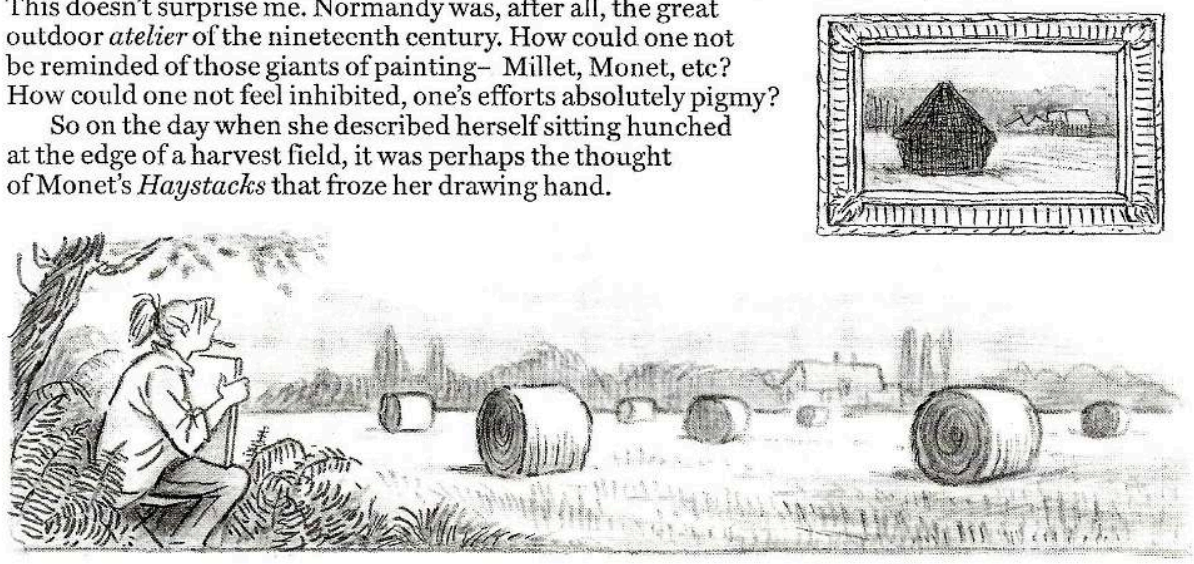

FiguRE 7. Posy Simmonds, Gemma Bovery. London: Jonathan Cape, 1999, p. 38. 

authentique, en imitant les intérieurs parfaits des magazines de décoration, le résultat reste peu probant. La planche qui rend compte de l'épisode enchaîne les plans figés, comme pour rappeler les pages de magazines. Mais l'image fixe signifie aussi l'échec de la copie sans vie, la nature morte dans un sens presque littéral, comme le confirme le commentaire très ironique de Joubert: «As the diary indicates, it was the assemblage of these objects that gave Gemma her happiest hours. No Old Dutch Master could have put more effort into the arrangement of nature morte » (36). L'effet de paralysie dans ces cases fait du passage une rupture avec l'art séquentiel: en renonçant au langage du mouvement qu'est la bande dessinée, les images évoquent un tableau culturellement inapproprié, sans valeur dans le récit et sans force évocatrice. Dans ces deux exemples, le chef-d'œuvre est un modèle auquel on n'échappe pas, mais qu'il faut savoir exploiter avec subtilité. On notera ici encore une pointe d'autodérision, car l'ironie pourrait se retourner contre le roman graphique, en suggérant que s'attaquer aux modèles serait toujours une entreprise hasardeuse. En même temps, et même si c'est son narrateur qui en assume l'initiative, Posy Simmonds défend aussi le droit qu'a l'auteur de roman graphique ou de bande dessinée d'utiliser le chef-d'œuvre, qu'il soit pictural ou littéraire et ce, de diverses manières.

effet, le positionnement culturel concerne aussi le roman graphique dans son ensemble, et les errements des personnages en la matière offrent un point de départ à ces questionnements. Au-delà de la satire et du traitement burlesque déjà évoqués, Posy Simmonds invente en parallèle son propre rapport à la littérature et à la culture. $\mathrm{Au}$ lieu de les considérer uniquement comme des modèles a priori inaccessibles aux codes d'écriture de la bande dessinée, elle explore les moyens de les intégrer en tant qu'objets culturels transférables d'un média à l'autre, que l'on peut et doit faire descendre de leur piédestal et ainsi rendre solubles dans le récit. Les références littéraires et artistiques qui appartiennent à la culture dite « légitime » émaillent le récit : outre l'hommage à Madame Bovary, le récit inclut plusieurs lettres dont la lecture précipite les événements, comme dans un roman épistolaire; le chien de Gemma s'appelle Carrington ; Joubert interprète certains intérieurs à la lumière de ses lectures, car l'un est «very Dickens» (21) et l'autre «Hogarthy»(19); on y voit des transpositions visuelles de tableaux de Monet et de Manet absorbées par le style graphique de Posy Simmonds; dans l'épilogue, la nouvelle voisine qui remplace les Bovery porte le nom prometteur de Jane Eyre. Certes, cet ancrage culturel très classique, voire consensuel, procure au récit la garantie que le propos appartient d'une manière ou d'une autre à la haute culture et ce, malgré la satire qui sous-tend le rapport au canon. Mais l'auteur parvient en parallèle à confirmer la légitimité de la bande dessinée en tant que medium, et en dehors d'une soumission au modèle, à la fois dans le rapport à la culture légitime et en dehors d'elle. Quel rapport ce roman graphique peut-il alors établir avec la culture classique, autrement que dans l'espoir de se voir accorder une légitimité?

Une partie de la réponse se trouve sans doute dans la façon dont Posy Simmonds s'attache à associer différentes catégories de culture, en ayant conscience des échelles de valeurs qui les distinguent, mais en évitant de faire de leur hiérarchisation le sujet principal de leur rencontre. Sa façon d'éluder la question des catégorisations entre culture savante et culture populaire consiste alors à mélanger les genres, les registres et les tonalités pour démontrer qu'ils contribuent à niveau égal à la construction du

Polysèmes, 24 | 2020 
sens dans le roman graphique. Ce mélange procède ainsi à un aplanissement des différences, non pas absolu mais circonstanciel et opéré dans le contexte de cette œuvre en particulier. Ainsi, bien qu'ancrée dans la culture classique, Gemma Bovery emprunte aussi à la culture populaire. Certains passages traitant de l'idylle entre Emma et Hervé tiennent du roman photo, médium que l'auteur utilise mais dont elle rappelle aussi la dimension potentiellement comique. À la page 52, dans une scène amoureuse où la foudre renforce les clichés, l'onomatopée, associée à un dessin plus épais, transporte soudain le lecteur dans l'univers des bandes dessinées sentimentales ${ }^{11}$, incursion confirmée page 67, où une case en forme de cœur encadre les amants enlacés. La couverture de l'œuvre, qui dévoile un portrait de Gemma, est un autre exemple du mélange des genres. Le décolleté plongeant de l'héroïne lourdement maquillée et parée de rouge, alors que le roman graphique est en noir et blanc, fait également référence à la bande dessinée sentimentale. En revanche, le cadre de ce portrait, dont les sculptures ornementales imitent le style victorien, annonce l'ancrage dans une autre époque et un univers sans doute plus littéraire. Cette tendance à l'hétérogénéité stylistique trouve aussi une incarnation dans les explorations de Gemma pour redécorer sa longère, ce que le narrateur souligne par les étiquettes qu'il attribue à ces diverses tentatives, mêlant les époques, les aires géographiques et même les langues. Selon lui Gemma commence par le «style agripauvre » (58), qui est constitué d'une collection d'objets rustiques, puis passe au style "Swedish Dangerous Liaisons»(58), plus élégant et caractérisé par un tissu d'ameublement imitant la toile de Jouy.

Lorsque le mélange des genres affecte l'art pictural, la démarche se fait plus provocatrice. On pense à la copie transposée du Déjeuner sur l'herbe qui côtoie le dessin de paniers offrant à la vente saucisse de Morteau et foie de veau (28). Citons enfin un passage qui superpose la satire à l'inspiration poétique ${ }^{12}$, mélange audacieux s'il en est, et sans que l'une ne vienne exclure l'autre. Dans la planche relatant la rencontre entre Joubert et Gemma à la boulangerie, la passion pour le pain que Joubert exhibe dans ce contexte parait disproportionnée. L'anthropomorphisme quelque peu grotesque qu'il accorde à la miche de pain, qu'il porte dans ses bras comme un enfant ou un objet sacré avec une mine inspirée, complète le portrait de ce personnage facilement ridiculisé par ses prétentions. Au-delà du traitement comique du personnage, Posy Simmonds vise sans doute les Français en général et leur perception du pain comme un objet culturel majeur (figure 8) :

She was right. I make real bread, full of taste and texture, not white factory stuff. And if I do nothing else in my life but perpetuate the love of good honest bread, then I shall be content. Bread for me is a passion.

It is our patrimony. To hold bread in one's hands, to feel

its surface - each crest, ridge, valley, fissure, crevasse, is to feel the earth, the primeval crust where life began. It is to bathe one's senses in the colour and smell of ripened corn.

It is to taste sunlight. Nothing

is more natural, more humble

than bread. Nothing is

more sensual.

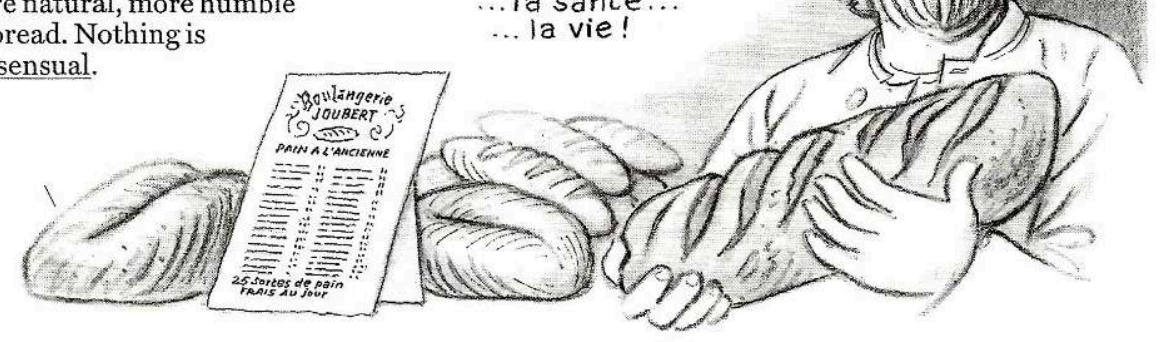

FiguRE 8. Posy Simmonds, Gemma Bovery. London: Jonathan Cape, 1999, p. 33. 

accompagne l'image les accents d'une voix qui se veut poétique, dans la mesure où l'objet magnifié par les sens devient support de l'imaginaire, révélant ici des merveilles insoupçonnées sous une apparente banalité. Aussi, cette esthétisation du pain, que Joubert voit comme une surface montagneuse faite de «crest, ridge, valley, fissure, crevasse ", n'est pas sans rappeler le poème de Francis Ponge qui observe la pâte à pain «façonnée en vallées, crêtes, ondulations, crevasses » (Ponge 46).

Ainsi Posy Simmonds invente-elle sa propre complexité, par des rapprochements incongrus, un goût pour l'éclectisme et la fusion entre culture populaire et culture classique. Par la satire et le traitement comique, mais aussi le retournement de situation, les clins d'œil, voire les pirouettes finales (on apprend à la dernière page que le vrai nom de Charlie est Cyril, ce qui met un terme à la malédiction littéraire et recolore tout le récit a posteriori), par ses manipulations ludiques de l'image et des mots, Posy Simmonds construit puis efface la limite entre les domaines de l'original et de l'ordinaire, entre l'imitation plate et la copie qui se distingue. Ce roman graphique qui ne se laisse pas aisément catégoriser revendique son appartenance à une généalogie littéraire mais sans se présenter uniquement comme un hypertexte. Il semble donc qu'à travers Gemma Bovery «la bande dessinée tire [pleinement] parti de sa situation secondaire, marginale, pour parasiter la tradition littéraire »(Garric), mais aussi et surtout s'autonomiser et s'affirmer comme une copie originale. La preuve en est que Gemma Bovery a également fait l'objet d'adaptations, le roman graphique ayant d'abord été transposé en film en 2014 par Anne Fontaine, puis en feuilleton radiophonique pour France Culture en 2018 par Lili Sztajn.

\section{BIBLIOGRAPHIE}

Baetens, Jan. « Littérature et bande dessinée : enjeux et limites ». Cahiers de Narratologie 16 (2009) (dernière consulation le 10 octobre 2020).

Baetens, Jan, Hugo Frey \& Stephen E. Tabachnick (eds.). The Cambridge History of the Graphic Novel. Cambridge: Cambridge UP, 2018.

Decout, Maxime. Qui a peur de l'imitation? Paris : Éditions de Minuit, 2017.

Dürrenmatt, Jacques. Bande dessinée et littérature. Paris : Classiques Garnier, 2013.

Gallice, Bruno. « Rémanence de Madame Bovary dans l'édition illustrée ». Flaubert 12 (2014)

(dernière consultation le 27 novembre 2020).

Garric, Henri. « Ce que la bande dessinée pense de la littérature : à propos de Gemma Bovery de Posy Simmonds ». neuvièmeart 2.0 (2014) (dernière consultation le 27 novembre 2020).

Gravett, Paul. So British ! L'Art de Posy Simmonds. Paris : Denoël, 2019.

Groensteen, Thierry. Système de la bande dessinée. Paris : PUF, 2011.

Hatfield, Charles. Alternative Comics: An Emerging Literature. Jackson: Mississippi UP, 2005. 
Hutcheon, Linda. A Theory of Adaptation. New York: Routledge, 2006.

Leclerc, Yvan. « Gemma Bovery, c'est elle ». Philosophie magazine 15 (2013) : 50-51.

Pizzino, Christopher. Arresting Development: Comics at the Boundaries of Literature. Austin: Texas UP, 2016.

Ponge, Francis. Le Parti pris des choses (1942). Paris : Gallimard, 1997.

Queffélec, Christine. " "La vie imite rarement l'Art” : Gemma Bovery, entre Flaubert et Wilde ». Revue de littérature comparée 3.355 (2015) : 299-307.

Servin, Lucie. « Qui êtes-vous Posy Simmonds ? » Les cahiers de la BD 7 (2019) : 94-96.

Simmonds, Posy. Gemma Bovery. New York: Pantheon Books, 1999.

Simmonds, Posy. Tamara Drewe. London: Jonathan Cape, 2007.

Simmonds, Posy. Cassandra Darke. London: Jonathan Cape, 2018.

Simmonds, Posy. True Love. London: Jonathan Cape, 1981.

Stein, Daniel \& Jan-Noël Thon. From Comic Strips to Graphic Novels: Contributions to the Theory and History of Graphic Narrative. Berlin: De Gruyter, 2013.

Wargny, Danielle. « Emma, version BD, version GB ». Le Bovarysme et la littérature de langue anglaise. Yvan Leclerc et Nicole Terrien (dir.). Mont-Saint-Aignan : PU de Rouen et du Havre, 2004, 209-218.

\section{NOTES}

1. Sur ces questions, voir Groensteen 8-14.

2. Joubert, qui est boulanger à Bailleville en Normandie, raconte l'histoire d'une jeune Anglaise fraîchement installée au village en relatant non seulement leurs rares rencontres, mais aussi les moments où il l'a suivie et épiée. Une autre source majeure de son récit est constituée des journaux intimes de Gemma que Joubert a subtilisés à son mari après la mort de celle-ci. Enfin, afin de combler les lacunes de son récit, Joubert extrapole et fantasme à partir des carnets de la jeune femme.

3. La critique littéraire et la critique journalistique considèrent Gemma Bovery soit comme une bande dessinée, soit comme un roman graphique, en raison probablement du lien noué avec le roman, de la multiplicité de ses sources et de la complexité de leur combinaison. Dans le présent article on emploiera généralement le terme de roman graphique, pour ces mêmes raisons. Posy Simmonds, quant à elle, voit volontiers Gemma Bovery comme un « roman illustré ».

4. Voir aussi l'article du Monde où sont repris les propos de Paul Gravett.

5. C'est ce que préconise Linda Hutcheon. Selon elle, l'évaluation de la qualité d'une adaptation ne devrait pas se fonder sur le degré de fidélité ou de trahison, mais bien plus sur les motivations de l'auteur qui adapte pour produire une œuvre finalement autonome (voir Hutcheon 20).

6. Voir à ce sujet Gravett : «L'une des règles tacites des comics modernes est de ne pas dépasser un certain nombre de mots par images. Aucun bédéaste avant Simmonds n'avait imaginé inclure des passages de texte "pour permettre au lecteur de se reposer des images" » (68).

7. Voir Maxime Decout: «Le plus souvent, il ne s'agit pas, pour l'écrivain, de simplement décalquer ou plagier, mais d'importer, d'adapter, de choisir ce qui est à même de nourrir son écriture ou son œuvre dans le texte d'un autre. C'est pour cette raison que l'imitation et l'influence ont souvent été décrites comme une sorte de digestion. Pour s'en convaincre, il suffit de se reporter à l'étymologie du mot "pastiche" qui nous donne une excellente leçon de 
modestie. Le mot est issu de l'italien "pasticcio" qui ne signifie rien moins que "pâté". C'est-à-dire un produit qui résulte de l'assemblage d'éléments variés » (7).

8. En outre, le style du dessin se rapproche ici de celui que l'on trouve dans certaines éditions illustrées de Madame Bovary. Peut-être Posy Simmonds cherche-t-elle là à rappeler que Madame Bovary a depuis longtemps entretenu des liens avec l'image et ainsi s'inscrire dans cette filiation. Sur les éditions illustrées de Madame Bovary, voir Bruno Gallice, "Rémanence de Madame Bovary dans l'édition illustrée ", Flaubert 12 (2014) (dernière consultation le 27 novembre 2020).

9. "Art imitates Life much more than Life imitates Art» est le paradoxe central sur lequel se fonde l'essai de Wilde «The Decay of Lying» (repris dans le recueil Intentions [1891]). Sur le lien entre Gemma Bovery et Wilde, voir Queffélec.

10. Il faut se souvenir ici des chroniques satiriques que Posy Simmonds a longtemps publiées dans le supplément littéraire du Guardian, où elle met en scène toute une galerie de personnages appartenant au monde littéraire dont elle dénonce les nombreux défauts et prétentions.

11. C'est un genre que Posy Simmonds a parodié dans True Love.

12. Posy Simmonds a eu d'autres occasions de tisser des liens entre image et poésie, comme par exemple lorsqu'elle a illustré un poème de Carol Ann Duffy pour le Guardian, en 2008.

\section{RÉSUMÉS}

D'un genre quasi inclassable, Gemma Bovery emprunte à la bande dessinée et au roman, voire au texte illustré, tout en intégrant collages et extraits littéraires. Le mode narratif est également original, car il conjugue le discours de la confession à celui de la biographie fabriquée. Au-delà de ces caractéristiques qui conduisent à des dispositifs visuels et narratifs peu communs en bande dessinée, l'idée de l'exception innerve l'œuvre à travers le rapport complexe au modèle, qu'il soit social, littéraire ou pictural. En jouant sur les différents sens et usages de l'image, Posy Simmonds construit des personnages graphiques à la fois banals et hors du commun : bien que soumis à un destin médiocre, car ils s'efforcent d'imiter des modèles sociaux-culturels préexistants sans parvenir à les égaler, ils sont transfigurés par le regard du narrateur qui les associe au roman de Flaubert. Mais le regard sans cesse satirique sur le narrateur et son récit remet en cause le statut de ces personnages supposément d'exception. Le geste de l'imitation est aussi un élément qui structure ce roman graphique: l'adaptation en bande dessinée prend la forme d'une transposition burlesque du chef-d'œuvre, mais donne en même temps naissance à une œuvre au fonctionnement autonome et qui accorde au roman flaubertien une présence graphique. Ainsi Posy Simmonds refuse-t-elle de soumettre son œuvre au modèle romanesque tout en tissant des liens avec lui. Plus généralement, le rapport à la culture vue comme légitime est un élément déterminant dans Gemma Bovery, car l'auteur y revendique son droit de mélanger les genres et les valeurs culturelles pour inventer sa propre singularité.

Posy Simmond's graphic work defies genre categorization, as it borrows from the language of comics, the novel and even illustrated texts, while it incorporates literary excerpts and various visual collages. Its narrative form - a pseudo biographical account embedded in a first-person discourse - is equally difficult to define. Beyond these features that allow for textual and visual arrangements rarely seen in comics, the idea of exception runs throughout the work in the form of a complex relationship to social, literary and visual models and references. Playing on the 
different meanings and functions of images leads Posy Simmonds to create characters that are both original and ordinary. Though they are trapped in their ordinary destiny, as their efforts to imitate pre-conceived social and cultural ideals are unsuccessful, they are transfigured by the narrator who associates them to Flaubert's Charles and Emma Bovary. Yet the constantly satirical viewpoint on the narrator and his account challenges the status of these supposedly exceptional characters. Moreover, imitating as a writing process structures the whole work: adapting Flaubert's masterpiece to the language of comics results in a burlesque transposition and yet an autonomous work, which also includes the novel as a graphic presence. Thus, Gemma Bovery succeeds in breaking away from Flaubert's novel as a model while being strongly connected to it. More generally, the relationship to high culture, which is central in the work, gives Posy Simmonds the opportunity to claim her right to mix different genres and cultural values to invent her own originality.

\section{INDEX}

Mots-clés : bande dessinée, roman graphique, adaptation, imitation, culture populaire oeuvrecitee Gemma Bovery

Keywords : comics, graphic novel, adaptation, imitation, popular culture

\section{AUTEURS}

\section{ORIANE MONTHÉARD}

Oriane Monthéard est maître de conférences à l'université de Rouen, où elle enseigne essentiellement la traduction. Son domaine de recherche principal est la poésie romantique et plus précisément John Keats. Elle a publié plusieurs articles sur la correspondance et la poésie de Keats et s'est notamment intéressée à l'importance de la marche dans les écrits de ce poète et épistolier, et à son rapport à la traduction. Elle a publié une monographie intitulée Keats et la rencontre (Grenoble : UGA Éditions, 2020) et codirige un ouvrage qui portera sur la traduction et l'adaptation du sonnet en Europe. Son autre domaine de recherche est le roman graphique de langue anglaise, l'hybridité du genre, son ambition et son esthétique. Elle publié un article sur les expériences autour de l'image dans From Hell d'Alan Moore et Eddie Campbell. 